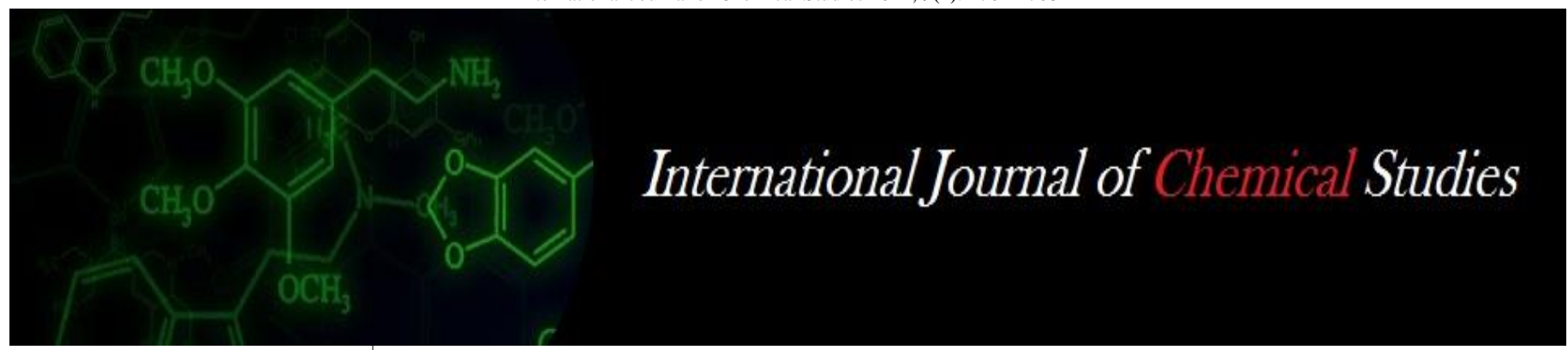

P-ISSN: 2349-8528

E-ISSN: 2321-4902

www.chemijournal.com

IJCS 2021; 9(1): 1761-1768

(C) 2021 IJCS

Received: 10-10-2020

Accepted: 16-12-2020

AD Falke

M.Sc. Department of

Agricultural Entomology,

Vasantrao Naik Marathwada

Krishi Vidyapeeth, Parbhani,

Maharashtra, India

\section{SK Patil}

M.Sc. Department of

Agricultural Entomology,

Vasantrao Naik Marathwada

Krishi Vidyapeeth, Parbhani,

Maharashtra, India

MM Sonkamble

M.Sc. Department of

Agricultural Entomology,

Vasantrao Naik Marathwada

Krishi Vidyapeeth, Parbhani,

Maharashtra, India
Corresponding Author:

SK Patil

M.Sc. Department of

Agricultural Entomology,

Vasantrao Naik Marathwada

Krishi Vidyapeeth, Parbhani,

Maharashtra, India

\section{Studies on ecofriendly approaches for the management of pulse beetle in chickpea}

\author{
AD Falke, SK Patil and MM Sonkamble
}

DOI: https://doi.org/10.22271/chemi.2021.v9.i1y.11481

\begin{abstract}
The present investigation was conducted to evaluate the different plant based products against pulse beetle (Callosobruchus chinensis Linnaeus) in chickpea in the laboratory of Department of Agricultural Entomology, VNMKV, Parbhani-431 402 (M.S.), India during Kharif 2019-20.

Among eight plant products evaluated, sweet flag rhizome powder @ 5\% was significantly found superior in reducing 100 per cent mortality, no seed damage, no seed weight loss with highest seed germination $(88.78 \%$ ) followed by neem seed kernel powder @ 5\% with higher adult mortality $(88.33 \%)$, less seed damage (15.33\%), less seed weight loss (4.71\%) and higher germination percentage (76.67\%) when seed stored for six month. Rest of the treatments viz., karanj seed powder@ $@$ \%, neem leaves powder @ 5\%, custard apple leaves powder @ 5\% and karanj leaves powder @ 5\% aslo showed less bruchid mortality $(75.00,65.00,53.33$, and $46.67 \%)$, seed damage $(28.67,42.00,51.33$ and $54 \%)$, weight loss $(18.67,20.07,26.13$, and $34.79 \%)$ and germination $(62.67,62.00,58.67$, and 53\%). The seed treated with turmeric rhizome powder @ 5\% was observed less effective in adult mortality $(36.67 \%)$, seed damage (33\%), weight loss (38.48\%) and germination (44.67\%) followed by tulsi leaves powder @ 5\% (51.67, 66.33, 37.82 and 52.00), respectively Whereas, untreated seed was recorded significantly maximum seed damage $(83.67 \%)$, seed weight loss $(47.33 \%)$ with no adult mortality and minimum germination $(37.00 \%)$ when it was stored for six month.
\end{abstract}

Keywords: Chickpea, plant powder, pulse beetle, adult mortality, seed damage, weight loss, germination

\section{Introduction}

Chickpea (Cicer arietinum L.) is considered as "King of Pulses" and is commonly known as "Bengal gram or Chana", belongs to family Fabaceae. Chickpea is second largest grown food legume of world. It is one of the important rabi pulse crop has been grown in India, Pakistan and a part of Africa. Among the pulses, chickpea is valued for its nutritive seeds with high protein content i.e. 25.3-28.9 per cent, 38- 59 per cent carbohydrate, 3 per cent fibre, 4.8-5.5 per cent oil, 3 per cent ash, 0.2 per cent calcium and 0.3 per cent phosphorus, after dehulling (Hulse, 1991) ${ }^{[7]}$. Beside its nutritional importance, chickpea has the medicinal property, malic acid and oxalic acid secreted by plant parts of this crop are prescribed for intestinal disorders. In India chickpea, was grown on 10.76 million hectares area with production 9.12 million tonnes and productivity was $969 \mathrm{~kg} / \mathrm{ha}$. Madhya Pradesh, Uttar Pradesh, Rajasthan, Maharashtra and Andhra Pradesh are the important chickpea producing states in India. The area under chickpea in Maharashtra is 18.48 lakh ha with production 18.81 lakh tonnes with the productivity $1018 \mathrm{~kg} / \mathrm{ha}$ and in Marathwada 8.70 lakh ha, production 9.18 lakh tonnes and productivity was $1027 \mathrm{~kg} /$ ha during 2017 (Anonymous, 2020) ${ }^{[1]}$.

Post-harvest losses of chickpea are very high in farmer storage conditions. The genus Callosobruchus attacks grain legumes during both pre and post-harvest stages all over the world. Pulse beetle, $C$. chinensis L. (Bruchidae: Coleoptera) is of significant importance as a major insect pest of stored chickpea (Rajasri et al., 2012) ${ }^{[11]}$. Pulse beetle causes not only quantitative but also qualitative losses like nutritive loss and make the chickpea unfit for marketing as well as consumption. The degree of damage varies with different kinds of legumes on the basis of exposure time, storage facilities and other factors associated with seeds (Srinivasan and Durairaj 2007) ${ }^{[15]}$. The stored chickpea attacked by pulse beetle which is major pest causing heavy losses to the tune of 10-60 per cent (Gupta and Kashyap 1971) ${ }^{[5]}$.

Control of storage pests by using synthetic chemicals has become a common practice among the farmers and stockholders. 
It is now widely known that chemical method has several problems, which include health hazards to the users and grain consumers. It causes residual toxicity, environmental pollution and development of pesticide resistance against bruchids. Sometimes persistent pesticides accumulate in the higher food chain of both wildlife and human and become concentrate by biomagnifications (Metcalf and Luckman, $1975)^{[10]}$. The traditional method of controlling storage pests by sun-drying is safer to human health and environment. But this method is laborious, time consuming, often expensive and requires suitable drying yard, when large volume of stored grain is involved. Moreover, it depends on favorable weather condition.

Recently, the use of different plants and their derivatives has appeared as an effective alternative to the poisonous chemical insecticides or the cumbersome traditional methods for the control of various insect pests of crops and storage. In the world, as many as 2400 plant species have been reported that have potential pesticide properties and biological activity against a wide range of pests (Grainge and Ahmed, 1988) ${ }^{[4]}$. Plant-derived materials are more readily biodegradable, some are less toxic to mammals, may be more selective in action and may retard the development of resistance. The main advantage is that they may be environmentally safe, less hazardous, economic and readily available, easily and cheaply produced by farmers and small-scale industries as crude, or partially purified extracts. Keeping in this view, the present investigation was carried out in considering hazards free botanicals aiming to assess the extent of damage of stored chickpea grains infested by pulse beetle.

\section{Material and Method}

The studies on "Eco-friendly management of pulse beetle in storage" was carried out under laboratory condition during 2019-20 at Department of Agricultural Entomology, College of Agriculture, Vasantrao Naik Marathwada Krishi Vidyapeeth, Parbhani-431402, Maharashtra (India).

\section{Seed}

Five hundred gram chickpea seeds were cleaned of straw, chaff, light grains and other impurities before testing. The grains were disinfected by keeping in the oven at $60{ }^{\circ} \mathrm{C}$ for 5 hours before keeping it for oviposition, development, loss in seed weight and seed infestation. Initially the moisture content of fresh seed was about 11.14 percent.

\section{Pulse beetle culture}

The initial pure culture of pulse beetle (Callosobruchus chinensis L.) as obtained from the office of Seed Processing Unit and the identification key of $C$. chinensis given by Raina (1970) ${ }^{[12]}$ was used. To initiate the culture, healthy grains of chickpea were kept in to $32 \mathrm{~cm} \times 22.5 \mathrm{~cm}$ size cylindrical jar and 10 pairs of bruchid adults were isolated and released into jar. The mouth of the container was covered with a muslin cloth secured firmly by rubber band. Fresh grains were provided periodically for the development of adults. After few days the new adults emergence, the bruchids were introduced into healthy chickpea seeds kept in series of cylindrical jars for building up a homogenous population. Density of population per jar was standardized to prevent overcrowding of adults which was reported by Sano (1967) ${ }^{[13]}$ to give rise to less reproductive active forms.

\section{Preparation of plant parts powder}

The plant part i.e. leaves, kernals and rhizomes of different botanicals was used for the present studies. The leaves of neem (Azadirachta indica A. Juss), tulsi (Oscimum basilicum L.), custard apple (Annona squamosa L.) and karanj (Pongamia glabra L) were collected and shade dried for a week then ground into powder. Kernels of neem and karanj was collected and made into powder using a grinder. Rhizomes of sweet flag (Acorus calamus L.) and turmeric (Curcuma longa L.) were procured from local market and made into bits and shade dried for a week then ground into powder.

\section{Methodology}

The above mentioned plant part powders were tested for their relative efficacy against C.chinensis at different storage periods. The experiment was carried out in a complete randomized design with three replication in treatments including untreated control (Table 1) in the laboratory (Temp. $26 \pm 2{ }^{\circ} \mathrm{C}$ and $75 \% \mathrm{RH}$ ). Chickpea variety BDNG 797 was used to carry out the experiment. The grains were sterilized at $60+2{ }^{\circ} \mathrm{C}$ for four hours in order to eliminate both apparent and hidden infestation, if any. Chickpea grains were weighed at the rate of $100 \mathrm{~g}$ each treatment per replication and kept in a medium sized (250 g capacity) plastic container. Five gm powder of each treatment was added to the respective containers and mixed thoroughly by shaking the containers. Twenty (20 pairs) newly emerged adults of pulse beetle, $C$. chinensis from the pure culture were released in each of the container containing treated grains and allowed to feed and oviposit. Plant products which showed promising results in this experiment were again evaluated after three months of their preparation by conducting another experiment following same procedure. The purpose of the experiment was to find any change in their effectiveness against the bruchids due to storage conditions.

\section{Observations}

The observation per cent adult mortality was calculated on the basis of the number of dead insects and also recorded per cent damaged seeds, per cent weight loss and germination percentage at 30,60, 90,120, 150 and 180 days after storage as per standard procedure.

$$
\begin{aligned}
& \text { Adult mortality }(\%)=\frac{\text { No. of live adults }- \text { No. of dead adults }}{\text { No. of live adults }} \times 100 \\
& \text { Seed damage }(\%)=\frac{\text { Initial No. of grains }- \text { Final No. of damaged grain }}{\text { Initial No. of grains }} \times 100 \\
& \text { Weight loss }(\%)=\frac{\text { Initial wt. of sound grains }- \text { Final wt. damaged grains }}{\text { Initial wt. of sound grains }} \times 100 \\
& \text { Germination }(\%)=\frac{\text { Number of grains germinated }}{\text { Total number of grains used }} \times 100
\end{aligned}
$$

\section{Statistical analysis}

The data obtained from different treatments were subjected to statistical analysis as per the statistical guidelines by Gomez and Gomez (1984) ${ }^{[3]}$. The results were transformed to arc sin values. The significance of treatment was tested by critical difference (C.D.) at 5 per cent level of significance for the comparison among the treatments, for which the marginal means of each treatment was considered.

\section{Results and Discussion}

The data collected with respect to per cent adult mortality of C. chinensis, per cent damaged seeds, per cent weight loss 
and germination percentage in chickpea seeds as influenced by various plant products at 30,60,90,120, 150 and 180 days after storage are presented in Table 1-4.

Table 1: Mortality of pulse beetle as influenced by different botanicals in chickpea seeds

\begin{tabular}{|c|c|c|c|c|c|c|c|c|}
\hline \multirow{2}{*}{ Sr. No. } & \multirow{2}{*}{ Treatments } & \multicolumn{7}{|c|}{ Percent adult mortality of pulse beetle (On number basis) } \\
\hline & & 30 DAS & 60 DAS & 90 DAS & 120 DAS & 150 DAS & 180 DAS & Mean \\
\hline 1. & Neem leaf powder $5 \%$ & $\begin{array}{c}91.67 \\
(73.22)\end{array}$ & $\begin{array}{c}86.67 \\
(68.58)\end{array}$ & $\begin{array}{c}81.67 \\
(64.65)\end{array}$ & $\begin{array}{c}75.00 \\
(60.00)\end{array}$ & $\begin{array}{c}70.00 \\
(56.79)\end{array}$ & $\begin{array}{c}65.00 \\
(53.73)\end{array}$ & $\begin{array}{c}78.33 \\
(62.26)\end{array}$ \\
\hline 2. & Tulsi leaf powder $5 \%$ & $\begin{array}{c}71.67 \\
(57.84)\end{array}$ & $\begin{array}{c}65.00 \\
(53.73)\end{array}$ & $\begin{array}{c}63.33 \\
(52.73)\end{array}$ & $\begin{array}{c}56.67 \\
(48.83) \\
\end{array}$ & $\begin{array}{c}51.67 \\
(45.96)\end{array}$ & $\begin{array}{c}45.00 \\
(42.13)\end{array}$ & $\begin{array}{c}58.89 \\
(50.12)\end{array}$ \\
\hline 3. & Custard apple leaf powder $5 \%$ & $\begin{array}{c}80.00 \\
(63.43)\end{array}$ & $\begin{array}{c}76.67 \\
(61.12)\end{array}$ & $\begin{array}{c}71.67 \\
(57.84)\end{array}$ & $\begin{array}{c}65.00 \\
(53.73)\end{array}$ & $\begin{array}{c}61.67 \\
(51.75)\end{array}$ & $\begin{array}{c}53.33 \\
(46.91)\end{array}$ & $\begin{array}{c}68.06 \\
(55.58)\end{array}$ \\
\hline 4. & Karanj leaf powder $5 \%$ & $\begin{array}{c}76.67 \\
(61.12)\end{array}$ & $\begin{array}{c}68.33 \\
(55.76)\end{array}$ & $\begin{array}{c}63.33 \\
(52.73)\end{array}$ & $\begin{array}{c}58.33 \\
(49.80)\end{array}$ & $\begin{array}{c}53.33 \\
(46.91)\end{array}$ & $\begin{array}{c}46.67 \\
(43.09)\end{array}$ & $\begin{array}{c}61.11 \\
(51.42)\end{array}$ \\
\hline 5. & Neem seed kernel powder $5 \%$ & $\begin{array}{c}98.33 \\
(82.58)\end{array}$ & $\begin{array}{c}96.67 \\
(79.48)\end{array}$ & $\begin{array}{c}95.00 \\
(77.08)\end{array}$ & $\begin{array}{c}93.33 \\
(75.04)\end{array}$ & $\begin{array}{c}90.00 \\
(71.57)\end{array}$ & $\begin{array}{c}88.33 \\
(70.03)\end{array}$ & $\begin{array}{c}93.61 \\
(75.36)\end{array}$ \\
\hline 6. & Karanj seed powder $5 \%$ & $\begin{array}{c}95.00 \\
(77.08)\end{array}$ & $\begin{array}{c}90.00 \\
(71.57)\end{array}$ & $\begin{array}{c}85.00 \\
(67.21)\end{array}$ & $\begin{array}{c}81.67 \\
(64.65)\end{array}$ & $\begin{array}{c}80.00 \\
(63.43)\end{array}$ & $\begin{array}{c}75.00 \\
(60.00)\end{array}$ & $\begin{array}{c}84.44 \\
(66.77)\end{array}$ \\
\hline 7. & Turmeric rhizome powder $5 \%$ & $\begin{array}{c}53.33 \\
(46.91)\end{array}$ & $\begin{array}{c}48.33 \\
(44.04)\end{array}$ & $\begin{array}{c}43.33 \\
(41.17)\end{array}$ & $\begin{array}{c}40.00 \\
(39.23)\end{array}$ & $\begin{array}{c}38.33 \\
(38.25)\end{array}$ & $\begin{array}{c}36.67 \\
(37.27)\end{array}$ & $\begin{array}{c}43.33 \\
(41.17)\end{array}$ \\
\hline 8. & Sweet flag rhizome powder $5 \%$ & $\begin{array}{l}100.00 \\
(90.00)\end{array}$ & $\begin{array}{l}100.00 \\
(90.00)\end{array}$ & $\begin{array}{l}100.00 \\
(90.00)\end{array}$ & $\begin{array}{l}100.00 \\
(90.00)\end{array}$ & $\begin{array}{l}100.00 \\
(90.00)\end{array}$ & $\begin{array}{l}100.00 \\
(90.00)\end{array}$ & $\begin{array}{l}100.00 \\
(90.00)\end{array}$ \\
\hline 9. & Untreated check & $\begin{array}{c}0.00 \\
(0.00)\end{array}$ & $\begin{array}{c}0.00 \\
(0.00)\end{array}$ & $\begin{array}{c}0.00 \\
(0.00)\end{array}$ & $\begin{array}{c}0.00 \\
(0.00)\end{array}$ & $\begin{array}{c}0.00 \\
(0.00)\end{array}$ & $\begin{array}{c}0.00 \\
(0.00)\end{array}$ & $\begin{array}{c}0.00 \\
(0.00)\end{array}$ \\
\hline & S.E. $(\mathrm{m}) \pm$ & 2.986 & 4.508 & 1.329 & 1.571 & 1.469 & 0.781 & 0.537 \\
\hline & C.D. at $5 \%$ & 8.951 & 11.675 & 3.983 & 4.709 & 4.404 & 2.341 & 1.611 \\
\hline & C.V. (\%) & 8.338 & 9.379 & 4.113 & 5.081 & 4.922 & 2.747 & 1.700 \\
\hline
\end{tabular}

Figures in parentheses are arcsine transformed values, DAS-Days after storage

Table 2: Seed damage by pulse beetle as influenced by different botanicals in chickpea

\begin{tabular}{|c|c|c|c|c|c|c|c|c|}
\hline \multirow{2}{*}{ Sr. No. } & \multirow{2}{*}{ Treatments } & \multicolumn{7}{|c|}{ Per cent seed damage (on number basis) } \\
\hline & & 30 DAS & 60 DAS & 90 DAS & 120 DAS & 150 DAS & 180 DAS & Mean \\
\hline 1. & Neem leaf powder $5 \%$ & $\begin{array}{c}4.67 \\
(12.48)\end{array}$ & $\begin{array}{c}15.00 \\
(22.79)\end{array}$ & $\begin{array}{c}22.00 \\
(27.97)\end{array}$ & $\begin{array}{c}32.67 \\
(34.86)\end{array}$ & $\begin{array}{c}37.00 \\
(37.46)\end{array}$ & $\begin{array}{c}42.00 \\
(40.40)\end{array}$ & $\begin{array}{c}25.56 \\
(30.37)\end{array}$ \\
\hline 2. & Tulsi leaf powder $5 \%$ & $\begin{array}{c}7.33 \\
(15.71)\end{array}$ & $\begin{array}{c}18.00 \\
(25.10) \\
\end{array}$ & $\begin{array}{c}33.00 \\
(35.06) \\
\end{array}$ & $\begin{array}{c}46.00 \\
(42.71) \\
\end{array}$ & $\begin{array}{c}52.67 \\
(46.53) \\
\end{array}$ & $\begin{array}{c}66.33 \\
(54.53) \\
\end{array}$ & $\begin{array}{c}37.22 \\
(37.60) \\
\end{array}$ \\
\hline 3. & Custard apple leaf powder $5 \%$ & $\begin{array}{c}6.00 \\
(14.18) \\
\end{array}$ & $\begin{array}{c}15.67 \\
(23.32) \\
\end{array}$ & $\begin{array}{c}25.00 \\
(30.00) \\
\end{array}$ & $\begin{array}{c}40.00 \\
(39.23) \\
\end{array}$ & $\begin{array}{c}48.00 \\
(43.85) \\
\end{array}$ & $\begin{array}{c}51.33 \\
(45.76) \\
\end{array}$ & $\begin{array}{c}31.00 \\
(33.83) \\
\end{array}$ \\
\hline 4. & Karanj leaf powder $5 \%$ & $\begin{array}{c}7.00 \\
(15.34)\end{array}$ & $\begin{array}{c}17.33 \\
(24.60)\end{array}$ & $\begin{array}{c}23.33 \\
(28.88)\end{array}$ & $\begin{array}{c}36.67 \\
(37.27)\end{array}$ & $\begin{array}{c}45.00 \\
(42.13)\end{array}$ & $\begin{array}{c}54.00 \\
(47.29)\end{array}$ & $\begin{array}{c}30.56 \\
(33.56)\end{array}$ \\
\hline 5. & Neem seed kernel powder $5 \%$ & $\begin{array}{c}2.67 \\
(9.40) \\
\end{array}$ & $\begin{array}{c}8.00 \\
(16.43) \\
\end{array}$ & $\begin{array}{c}6.67 \\
(14.96) \\
\end{array}$ & $\begin{array}{c}10.67 \\
(19.06) \\
\end{array}$ & $\begin{array}{c}13.33 \\
(21.42) \\
\end{array}$ & $\begin{array}{c}15.33 \\
(23.05) \\
\end{array}$ & $\begin{array}{c}9.44 \\
(17.90) \\
\end{array}$ \\
\hline 6. & Karanj seed powder $5 \%$ & $\begin{array}{c}3.33 \\
(10.52) \\
\end{array}$ & $\begin{array}{c}13.67 \\
(21.70) \\
\end{array}$ & $\begin{array}{c}16.00 \\
(23.58)\end{array}$ & $\begin{array}{c}20.67 \\
(27.04) \\
\end{array}$ & $\begin{array}{c}34.33 \\
(35.87) \\
\end{array}$ & $\begin{array}{c}28.67 \\
(32.37) \\
\end{array}$ & $\begin{array}{c}19.44 \\
(26.17) \\
\end{array}$ \\
\hline 7. & Turmeric rhizome powder $5 \%$ & $\begin{array}{c}10.67 \\
(19.06)\end{array}$ & $\begin{array}{c}33.67 \\
(35.47) \\
\end{array}$ & $\begin{array}{c}54.00 \\
(47.29) \\
\end{array}$ & $\begin{array}{c}63.67 \\
(52.93) \\
\end{array}$ & $\begin{array}{c}78.33 \\
(62.26) \\
\end{array}$ & $\begin{array}{c}63.33 \\
(52.73) \\
\end{array}$ & $\begin{array}{c}50.61 \\
(45.35) \\
\end{array}$ \\
\hline 8. & Sweet flag rhizome powder $5 \%$ & $\begin{array}{c}0.00 \\
(0.00)\end{array}$ & $\begin{array}{c}0.00 \\
(0.00)\end{array}$ & $\begin{array}{c}0.00 \\
(0.00)\end{array}$ & $\begin{array}{c}0.00 \\
(0.00)\end{array}$ & $\begin{array}{c}0.00 \\
(0.00)\end{array}$ & $\begin{array}{c}0.00 \\
(0.00)\end{array}$ & $\begin{array}{c}0.00 \\
(0.00)\end{array}$ \\
\hline 9. & Untreated check & $\begin{array}{c}21.33 \\
(27.51)\end{array}$ & $\begin{array}{c}53.67 \\
(47.10)\end{array}$ & $\begin{array}{c}61.00 \\
(51.35)\end{array}$ & $\begin{array}{c}75.00 \\
(60.00)\end{array}$ & $\begin{array}{c}86.33 \\
(68.30)\end{array}$ & $\begin{array}{c}83.67 \\
(66.16)\end{array}$ & $\begin{array}{c}63.50 \\
(52.83)\end{array}$ \\
\hline & S.E. $(\mathrm{m}) \pm$ & 0.792 & 0.733 & 0.644 & 0.692 & 1.227 & 0.878 & 0.344 \\
\hline & C.D. at $5 \%$ & 2.376 & 2.197 & 1.931 & 2.076 & 3.679 & 2.634 & 1.031 \\
\hline & C.V. $(\%)$ & 9.976 & 5.280 & 3.875 & 3.448 & 5.345 & 3.779 & 1.932 \\
\hline
\end{tabular}

Figures in parentheses are arcsine transformed values, DAS-Days after storage

Table 3: Loss in weight due to pulse beetle as influenced by different botanicals in chickpea seeds

\begin{tabular}{|c|c|c|c|c|c|c|c|c|}
\hline \multirow{2}{*}{ Sr. No. } & \multirow{2}{*}{ Treatments } & \multicolumn{7}{|c|}{ Percent loss in weight (On weight basis) } \\
\hline & & 30 DAS & 60 DAS & 90 DAS & 120 DAS & $150 \mathrm{DAS}$ & 180 DAS & Mean \\
\hline 1. & Neem leaf powder $5 \%$ & $\begin{array}{c}1.59 \\
(7.25)\end{array}$ & $\begin{array}{c}4.43 \\
(12.15)\end{array}$ & $\begin{array}{c}9.80 \\
(18.25)\end{array}$ & $\begin{array}{c}15.18 \\
(22.93)\end{array}$ & $\begin{array}{c}18.05 \\
(25.14)\end{array}$ & $\begin{array}{c}20.07 \\
(26.62)\end{array}$ & $\begin{array}{c}11.52 \\
(19.84) \\
\end{array}$ \\
\hline 2. & Tulsi leaf powder $5 \%$ & $\begin{array}{c}3.65 \\
(11.01)\end{array}$ & $\begin{array}{c}9.97 \\
(18.40)\end{array}$ & $\begin{array}{c}18.61 \\
(25.56)\end{array}$ & $\begin{array}{c}26.69 \\
(31.11)\end{array}$ & $\begin{array}{c}35.10 \\
(36.33)\end{array}$ & $\begin{array}{c}37.82 \\
(37.95)\end{array}$ & $\begin{array}{c}21.97 \\
(27.95)\end{array}$ \\
\hline 3. & Custard apple leaf powder $5 \%$ & $\begin{array}{c}2.44 \\
(8.98) \\
\end{array}$ & $\begin{array}{c}4.74 \\
(12.57) \\
\end{array}$ & $\begin{array}{c}12.04 \\
(20.30) \\
\end{array}$ & $\begin{array}{c}18.53 \\
(25.49) \\
\end{array}$ & $\begin{array}{c}20.95 \\
(27.24) \\
\end{array}$ & $\begin{array}{c}26.13 \\
(30.74) \\
\end{array}$ & $\begin{array}{c}14.14 \\
(22.09) \\
\end{array}$ \\
\hline 4. & Karanj leaf powder $5 \%$ & $\begin{array}{c}3.62 \\
(10.97) \\
\end{array}$ & $\begin{array}{c}8.74 \\
(17.19) \\
\end{array}$ & $\begin{array}{c}15.53 \\
(23.21) \\
\end{array}$ & $\begin{array}{c}23.65 \\
(29.10) \\
\end{array}$ & $\begin{array}{c}24.97 \\
(29.98) \\
\end{array}$ & $\begin{array}{c}34.79 \\
(36.15) \\
\end{array}$ & $\begin{array}{c}18.55 \\
(25.51) \\
\end{array}$ \\
\hline 5. & Neem seed kernel powder $5 \%$ & $\begin{array}{c}0.77 \\
(5.02) \\
\end{array}$ & $\begin{array}{c}1.20 \\
(6.28)\end{array}$ & $\begin{array}{c}1.93 \\
(7.99) \\
\end{array}$ & $\begin{array}{c}2.89 \\
(9.79) \\
\end{array}$ & $\begin{array}{c}4.16 \\
(11.77) \\
\end{array}$ & $\begin{array}{c}4.73 \\
(12.56)\end{array}$ & $\begin{array}{c}2.61 \\
(9.30)\end{array}$ \\
\hline
\end{tabular}




\begin{tabular}{|c|c|c|c|c|c|c|c|c|}
\hline 6. & Karanj seed powder $5 \%$ & $\begin{array}{c}1.31 \\
(6.56)\end{array}$ & $\begin{array}{c}4.28 \\
(11.94)\end{array}$ & $\begin{array}{c}8.11 \\
(16.55)\end{array}$ & $\begin{array}{c}12.93 \\
(21.07)\end{array}$ & $\begin{array}{c}15.85 \\
(23.46)\end{array}$ & $\begin{array}{c}18.67 \\
(25.60)\end{array}$ & $\begin{array}{c}10.19 \\
(18.62)\end{array}$ \\
\hline 7. & Turmeric rhizome powder $5 \%$ & $\begin{array}{c}6.06 \\
(14.26)\end{array}$ & $\begin{array}{c}17.13 \\
(24.45) \\
\end{array}$ & $\begin{array}{c}20.79 \\
(27.13) \\
\end{array}$ & $\begin{array}{c}26.98 \\
(31.29) \\
\end{array}$ & $\begin{array}{c}35.85 \\
(36.78) \\
\end{array}$ & $\begin{array}{c}38.48 \\
(38.34) \\
\end{array}$ & $\begin{array}{c}24.21 \\
(29.48) \\
\end{array}$ \\
\hline 8. & Sweet flag rhizome powder $5 \%$ & $\begin{array}{c}0.00 \\
(0.00)\end{array}$ & $\begin{array}{c}0.00 \\
(0.00)\end{array}$ & $\begin{array}{c}0.00 \\
(0.00) \\
\end{array}$ & $\begin{array}{c}0.00 \\
(0.00)\end{array}$ & $\begin{array}{c}0.00 \\
(0.00)\end{array}$ & $\begin{array}{c}0.00 \\
(0.00)\end{array}$ & $\begin{array}{c}0.00 \\
(0.00)\end{array}$ \\
\hline 9. & Untreated check & $\begin{array}{c}9.87 \\
(18.31)\end{array}$ & $\begin{array}{c}18.23 \\
(25.28)\end{array}$ & $\begin{array}{c}26.70 \\
(31.11)\end{array}$ & $\begin{array}{c}35.24 \\
(36.42)\end{array}$ & $\begin{array}{c}41.90 \\
(40.34)\end{array}$ & $\begin{array}{c}47.33 \\
(43.47)\end{array}$ & $\begin{array}{c}29.88 \\
(33.14)\end{array}$ \\
\hline & S.E. $(\mathrm{m}) \pm$ & 0.783 & 0.343 & 0.266 & 0.648 & 0.539 & 0.368 & 0.181 \\
\hline & C.D. at $5 \%$ & 2.346 & 1.028 & 0.796 & 1.944 & 1.617 & 1.104 & 0.543 \\
\hline & C.V. $(\%)$ & 4.923 & 4.169 & 2.435 & 4.880 & 3.639 & 2.284 & 1.518 \\
\hline
\end{tabular}

Figures in parentheses are arcsine transformed values, DAS-Days after storage

Table 4: Germination of chickpea seeds as influenced by different botanicals used for the management of pulse beetle

\begin{tabular}{|c|c|c|c|c|c|c|c|c|}
\hline \multirow{2}{*}{ Sr. No. } & \multirow{2}{*}{ Treatments } & \multicolumn{7}{|c|}{ Percent germination } \\
\hline & & 30 DAS & 60 DAS & 90 DAS & 120 DAS & 150 DAS & 180 DAS & Mean \\
\hline 1. & Neem leaf powder $5 \%$ & $\begin{array}{c}93.00 \\
(74.66)\end{array}$ & $\begin{array}{c}88.67 \\
(70.33)\end{array}$ & $\begin{array}{c}82.33 \\
(65.15)\end{array}$ & $\begin{array}{c}73.33 \\
(58.91)\end{array}$ & $\begin{array}{c}63.33 \\
(52.73)\end{array}$ & $\begin{array}{c}62.00 \\
(51.94)\end{array}$ & $\begin{array}{c}77.11 \\
(61.42)\end{array}$ \\
\hline 2. & Tulsi leaf powder $5 \%$ & $\begin{array}{c}84.67 \\
(66.95)\end{array}$ & $\begin{array}{c}84.33 \\
(66.68)\end{array}$ & $\begin{array}{c}71.67 \\
(57.84) \\
\end{array}$ & $\begin{array}{c}61.67 \\
(51.75)\end{array}$ & $\begin{array}{c}55.00 \\
(47.87)\end{array}$ & $\begin{array}{c}52.00 \\
(46.15)\end{array}$ & $\begin{array}{c}68.22 \\
(55.69) \\
\end{array}$ \\
\hline 3. & Custard apple leaf powder $5 \%$ & $\begin{array}{c}88.67 \\
(70.33)\end{array}$ & $\begin{array}{c}84.67 \\
(66.95)\end{array}$ & $\begin{array}{c}79.67 \\
(63.20)\end{array}$ & $\begin{array}{c}68.67 \\
(55.96)\end{array}$ & $\begin{array}{c}62.67 \\
(52.34)\end{array}$ & $\begin{array}{c}58.67 \\
(49.99)\end{array}$ & $\begin{array}{c}73.83 \\
(59.23)\end{array}$ \\
\hline 4. & Karanj leaf powder $5 \%$ & $\begin{array}{c}91.67 \\
(73.22)\end{array}$ & $\begin{array}{c}85.67 \\
(67.75)\end{array}$ & $\begin{array}{c}74.00 \\
(59.34)\end{array}$ & $\begin{array}{c}66.67 \\
(54.74)\end{array}$ & $\begin{array}{l}61.00 \\
51.35)\end{array}$ & $\begin{array}{c}53.00 \\
(46.72)\end{array}$ & $\begin{array}{c}72.00 \\
(58.05)\end{array}$ \\
\hline 5. & Neem seed kernel powder $5 \%$ & $\begin{array}{c}94.33 \\
(76.23) \\
\end{array}$ & $\begin{array}{c}91.67 \\
(73.22) \\
\end{array}$ & $\begin{array}{c}83.33 \\
(65.91) \\
\end{array}$ & $\begin{array}{c}80.33 \\
(63.67) \\
\end{array}$ & $\begin{array}{c}77.67 \\
(61.80) \\
\end{array}$ & $\begin{array}{c}76.67 \\
(61.12) \\
\end{array}$ & $\begin{array}{r}84.00 \\
(66.42) \\
\end{array}$ \\
\hline 6. & Karanj seed powder $5 \%$ & $\begin{array}{c}89.67 \\
(71.25) \\
\end{array}$ & $\begin{array}{c}84.33 \\
(66.68) \\
\end{array}$ & $\begin{array}{c}80.33 \\
(63.67) \\
\end{array}$ & $\begin{array}{c}75.00 \\
(60.00) \\
\end{array}$ & $\begin{array}{c}72.33 \\
(58.27) \\
\end{array}$ & $\begin{array}{c}62.67 \\
(52.34) \\
\end{array}$ & $\begin{array}{r}77.39 \\
(61.61) \\
\end{array}$ \\
\hline 7. & Turmeric rhizome powder $5 \%$ & $\begin{array}{c}79.00 \\
(62.73)\end{array}$ & $\begin{array}{c}73.00 \\
(58.69)\end{array}$ & $\begin{array}{c}65.00 \\
(53.73)\end{array}$ & $\begin{array}{c}61.00 \\
(51.35)\end{array}$ & $\begin{array}{c}54.00 \\
(47.29)\end{array}$ & $\begin{array}{c}44.67 \\
(41.94)\end{array}$ & $\begin{array}{c}62.78 \\
(52.40)\end{array}$ \\
\hline 8. & Sweet flag rhizome powder $5 \%$ & $\begin{array}{c}94.67 \\
(76.65) \\
\end{array}$ & $\begin{array}{c}91.33 \\
(72.88) \\
\end{array}$ & $\begin{array}{c}88.67 \\
(70.33) \\
\end{array}$ & $\begin{array}{c}86.67 \\
(68.58) \\
\end{array}$ & $\begin{array}{c}85.67 \\
(67.75) \\
\end{array}$ & $\begin{array}{c}85.67 \\
(67.75) \\
\end{array}$ & $\begin{array}{c}88.78 \\
(70.43) \\
\end{array}$ \\
\hline 9. & $\begin{array}{l}\text { Untreated } \\
\text { check }\end{array}$ & $\begin{array}{c}75.00 \\
(60.00)\end{array}$ & $\begin{array}{c}64.00 \\
(53.13)\end{array}$ & $\begin{array}{c}58.33 \\
(49.80)\end{array}$ & $\begin{array}{c}56.00 \\
(48.45)\end{array}$ & $\begin{array}{c}50.33 \\
(45.19)\end{array}$ & $\begin{array}{c}37.00 \\
(37.46)\end{array}$ & $\begin{array}{c}56.78 \\
(48.90)\end{array}$ \\
\hline & S.E. $(m) \pm$ & 1.366 & 1.371 & 0.967 & 1.133 & 0.875 & 0.787 & 0.515 \\
\hline & C.D. at $5 \%$ & 4.095 & 4.110 & 2.899 & 3.396 & 2.623 & 2.359 & 1.543 \\
\hline & C.V. $(\%)$ & 3.364 & 3.580 & 2.745 & 3.437 & 2.813 & 2.693 & 1.502 \\
\hline
\end{tabular}

Figures in parentheses are arcsine transformed values, DAS-Days after storage

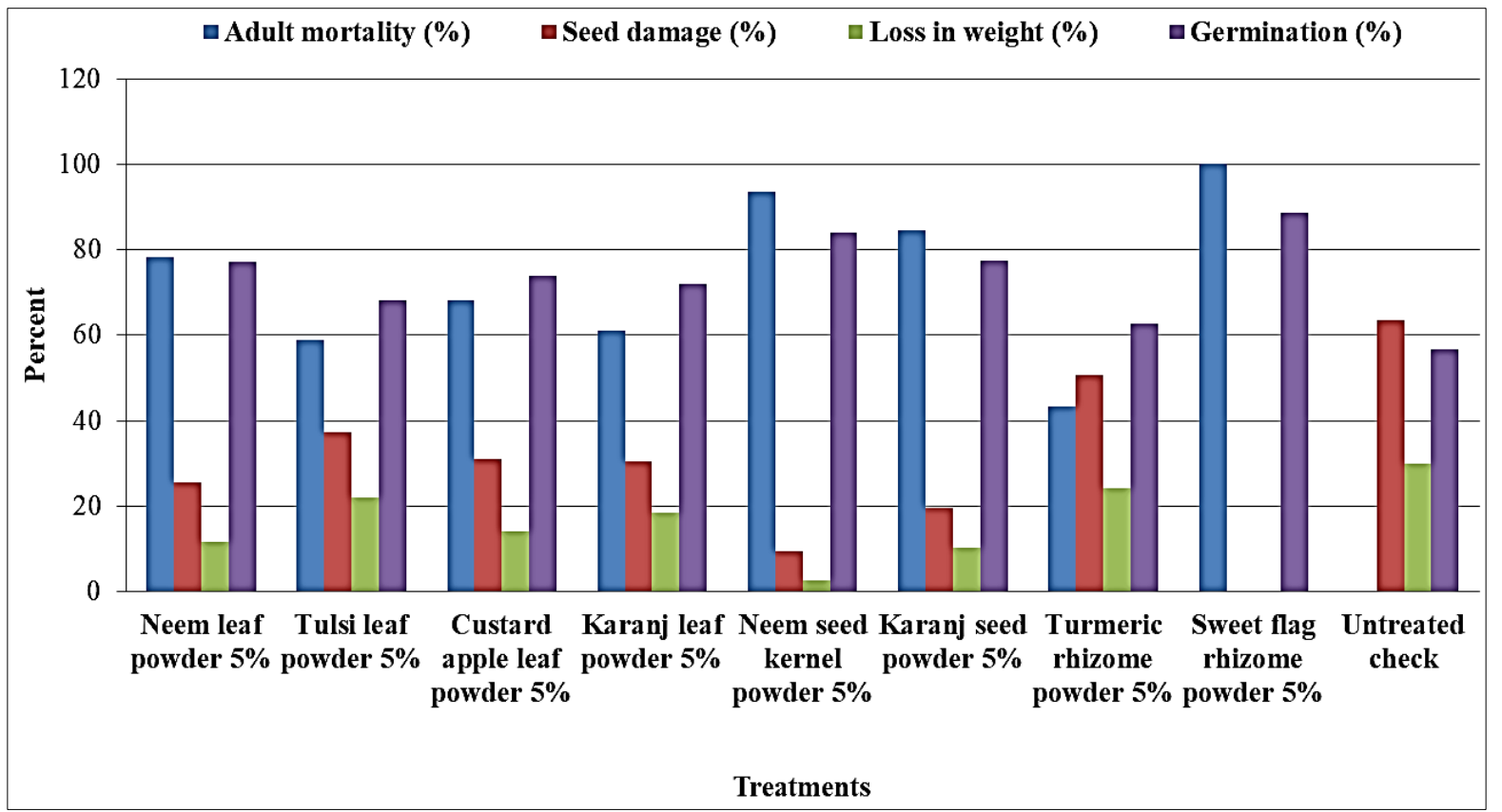

Fig 1: Adult mortality, seed damage, seed weight loss and germination of sorghum seeds as influenced by different botanicals used for the management of pulse beetle

\section{i) Adult mortality}

\section{At 30 days after storage}

At one month after storage, sweet flag rhizome powder @ 5\% was recorded 100 per cent adult mortality and it was at par with neem seed kernel powder@ $@$ \% (98.33\%). The next best treatments were karanj seed powder @ 5\% (95.00\%) followed by neem leaf powder @ 5\% (91.67\%), custard apple leaves powder 5\% (80\%), karanj leaves powder 5\% (76.67\%) and 
tulsi leaves powder @ 5\% (71.67\%). The significantly minimum mortality was observed in turmeric rhizome powder @ 5\% (53.33\%). No adult mortality was recorded in untreated check.

\section{At 60 days after storage}

All beetles were died in stored chickpea seed when treated with sweet flag rhizome powder @ 5\% (100\%). The next effective botanicals were neem seed kernel powder @ 5\% $(96.67 \%)$ and karanj seed powder @5\% (90.00\%). The beetles were significantly less susceptible to turmeric rhizome powder@ $@ 5 \%(48.33 \%)$ which was at par with tulsi leaves powder @ 5\% (65.00\%) and karanj leaves powder 5\% $(68.33 \%)$. Other botanicals also reduced pulse beetle infestation in the range of 86.67 to 76.67 per cent.

\section{At 90 days after storage}

At 90 DAS, Sweet flag rhizome powder @ 5\% was also significantly caused 100 per cent adult mortality followed by neem seed kernel powder @ 5\% (95.00\%) and karamj seed powder@5\% (85.00\%). The treatments viz., neem leaves powder@5\% custard apple leaves powder 5\% and karanj leaves powder 5\% was recorded mortality of 81.67, 71.67 and 63.33 per cent, respectively. Significantly less mortality adults were noticed in turmeric rhizome powder @ 5\% $(43.33 \%)$ followed by tulsi leaves powder @ 5\% (63.33\%).

\section{At 120 days after storage}

Similar trend was observed at 120 days after storage. However, sweet flag rhizome powder @ 5\% significantly caused hundred per cent mortality followed by 93.33 per cent in neem seed kernel powder @ 5\%.The next superior treatments was karanj seed powder @ 5\% and neem leaves powder@5\% that caused 81.67 and 75 per cent adult mortality, respectively. The significantly minimum mortality was observed in turmeric rhizome powder @ 5\% (40.00\%) followed by tulsi leaves powder@ @\% (56.67\%).

\section{At 150 days after storage}

Sweet flag rhizome powder @ 5\% was found to superior over other botanicals treatment by causing 100 per cent adult mortality followed by neem seed kernel powder @ 5\% (90\%). The next best treatment was karanj seed powder powder @ $5 \%$ and neem leaves powder powder $5 \%$ that caused $(80.00$ and $70.00 \%$, respectively). The treatment turmeric rhizome powder@ $5 \%(38.33 \%)$ was found significantly less effective in reducing the mortality followed by followed by tulsi leaves powder@5\%(51.67\%).

\section{At 180 days after storage}

Among different botanicals sweet flag rhizome powder@ $9 \%$ results with 100 per cent mortality of adult beetle at 180 days after storage,. The treatments i.e. neem seed kernel powder @ $5 \%(88.33 \%)$ and karanj seed powder @ 5\% (75.\%) found also effective to reduce adult population. The significantly minimum mortality was observed in turmeric rhizome powder @ 5\% (36.67\%) followed by tulsi leaves powder @ 5\% (45\%), karanj leaves powder 5\% (46.67\%), neem leaves powder $5 \%(65 \%)$ and custard apple leaves powder $5 \%$ $(53.33 \%)$ in six month stored chickpea seed.

\section{Mean adult mortality over 180 days stored chickpea}

Among all botanicals evaluated for their efficacy over 180 days of storage, the best treatments in order of efficacy was sweet flag rhizome powder @ 5\%, caused significantly maximum adult mortality $(100.00 \%)$ followed by neem seed kernel powder @ 5\% (93.61\%), karanj seed powder @ 5\% $(84.44 \%)$, neem leaves powder 5\% $(78.33 \%)$, custard apple leaves powder $5 \%(68.06 \%)$, karanj leaves powder $5 \%$ $(61.11 \%)$ and tulsi leaves powder @ 5\% (58.89\%). Whereas, C. chinensis was less susceptible to turmeric rhizome powder @\% (43.33\%).

The results of present investigation corroborated with the results of Kaur et al. (2019) [8] reported that sweet flag resulted in $64.49,84.44$ and 98.89 per cent mortality of the pulse beetle, $C$. chinensis at the highest dose ( $5 \mathrm{~g} / 100 \mathrm{~g}$ seeds) after 1,3 and 7 days of treatment, respectively. Neem was other highly effective product that accounted for $18.89,42.22$ and 63.33 per cent mortality of the beetle after the respective treatment intervals. Govindan and Nelson (2008) ${ }^{[2]}$ reported that the sweet flag powder caused less mortality of adults of C. chinensis. Similar to present study, Shukla et al. (2009) ${ }^{[14]}$ also reported that sweet flag rhizome powder caused 100 per cent mortality of $C$. chinensis. and also reported the bioefficacy of sweet flag powder with 100 per cent ovicidal activity and completely inhibiting adult emergence against pulse beetle infesting stored chickpea seeds. Khalequzzaman et al. (2009) ${ }^{[9]}$ showed the results the per cent mortality rates were recorded in the ranged of $26.66 \pm 12.01$ to 100 per cent for $C$. maculatus and $86.66 \pm 6.66$ to 100 per cent for $C$. chinensis when seed treated with $A$. indica seed kernel.

\section{ii) Seed damage}

\section{At 30 days after storage}

All the plant products treatments were found to be significantly reduced seed damage as against untreated control. The seeds treated with sweet flag rhizome powder @ $5 \%$ was recorded no damage by beetles at 30 DAS. The next best treatment was neem seed kernel powder @ 5\% (2.67\%) which was at par with karanj seed powder @ 5\% (3.33\%) followed by neem leaves powder @ 5\% (4.67\%), custard apple leaves powder 5\% (6.00\%), karanj leaves powder @ 5\% $(7.00 \%)$, tulsi leaves powder $5 \%(7.33 \%)$ and turmeric rhizome powder@5\% (10.67\%). The significantly maximum seed damage was noticed in untreated check $(21.33 \%)$.

\section{At 60 days after storage}

Among the different botanicals, no seed damage was recorded in sweet flag rhizome powder @ 5\% even at 60 DAS. The significantly next best promising treatment was neem seed kernel powder@ $@ 5 \%(8 \%)$ followed by karanj seed powder @ 5\% (13.67\%). While, significantly more damage to seeds was observed in turmeric rhizome powder@ $@$ \% (33.67\%) followed by tulsi leaves powder @ 5\% (18\%) which were at par with untreated check $(53.67 \%)$.

\section{At 90 days after storage}

The seed treated with sweet flag rhizome powder @ 5\% was found toxic effect by protecting seeds without damage upto 90 DAS also, followed by neem seed kernel powder @ 5\% $(6.67 \%)$ and karanj seed powder 5\% (16\%). However, more damage to seeds was observed in turmeric rhizome powder @ $5 \%(54 \%)$ followed by tulsi leaves powder @ 5\% (33\%) and maximum damage was recorded in untreated check $(61 \%)$.

\section{At 120 days after storage}

Significantly no damage to seed was observed in sweet flag rhizome powder@5\%. Significantly maximum damage was noticed in untreated check $(75 \%)$ followed by turmeric 
rhizome powder @ 5\% (63.67\%) and tulsi leaves powder 5\% $(46 \%)$. Rest of the botanical was recorded seed damage in the range of 10.67 to 40.00 per cent.

\section{At 150 days after storage}

No seed damage was observed in sweet flag rhizome powder @ 5\% at 150 days after storage. The treatment neem seed kernel powder@5\% (13.33\%) was found next effective against pulse beetle. Other treatments were recorded seed damage in between 34.33 and 78.33 per cent. Similarly, more seed damage caused by bruchids in untreated check ( $86.33 \%)$.

\section{At 180 days after storage}

Among different botanicals evaluated for their efficacy against $C$. chinensis, sweet flag rhizome powder @ 5\% did not allow the pest to damage the seed. While significantly minimum damage was observed in neem seed kernel powder @ 5\% (15.33\%). The treatments viz., karanj seed powder @ $5 \%$, neem leaves powder 5\%, custard apple leaves powder $5 \%$, karanj leaves powder $5 \%$, tulsi leaves powder, turmeric rhizome powder@5\% and seed infestation caused by pulse beetle was 28.67, 42.00, 51.33, 54.00, 63.33 and 66.33 per cent, respectively when seed stored for 6 month period. Significantly maximum damage was recorded in untreated check $(83.67 \%)$.

\section{Mean seed damage due to $C$. chinensis over 180 days of storage}

Sweet flag rhizome powder @ 5\% protected seeds from $C$. chinensis with 100 per cent protection followed by neem seed kernel powder @ 5\% (9.44\%) showing best results. Rest of the treatments in order of their efficacy was karanj seed powder $5 \%(19.44 \%)>$ neem leaves powder $5 \%(25.56 \%)>$ karanj leaves powder $5 \%(30.56 \%)>$ custard apple leaves powder 5\% (31\%) > tulsi leaves powder @ 5\% (37.22\%)> turmeric rhizome powder @ 5\% (50.61\%)> untreated check $(63.50 \%)$.

These results are supported with the finding reported by Tripathi et al. (2006) ${ }^{[17]}$ who noted that mixing of seeds with neem leaf powder has been the effective control measure of pest infestation. of the pulse beetle, $C$. chinensis L. in stored pigeon pea (Cajanus cajan L.). Tesema et al. (2015) [16] reported that all the tested locally available treatment (cow dung ash, leaf powder of neem and leaf powder of basil) were found to be effective in reducing the damage inflicted by bruchid, $C$. chinensis compared to the control.

\section{iii) Seed weight loss}

\section{At 30 days after storage}

Seed weight loss due to $C$. chinensis in untreated check was maximum $(9.87 \%)$ as compared to the seed treated with different plant products. No weight loss was recorded in seeds treated with sweet flag rhizome powder @ 5\%. Significantly next best treatment was neem seed powder @ 5\% (0.77\%) followed by karanj seed powder $5 \%(1.31 \%)$, neem leaves powder $5 \%(1.59 \%)$, custard apple leaves powder $5 \%(2.44 \%)$ and karanj leaves powder 5\% (3.62\%), tulsi leaves powder @ $5 \%(3.65 \%)$ and turmeric rhizome @ 5\% (6.06\%) at 30 days after storage.

\section{At 60 days after storage}

There was no seed weight loss when chickpea seeds treated with sweet flag rhizome powder @ 5\%. The next promising treatment was neem seed powder 5\% (1.20\%). The treatments viz., karanj seed powder@5\%, neem leaves powder 5\%, custard apple leaves powder 5\%, karanj leaves powder $5 \%$ and tulsi leaves powder @ 5\% was recorded of 4.28, 4.43, 4.74, 8.74 and 9.97 per cent, respectively. Significantly maximum seed weight loss was noticed in untreated check $(18.23 \%)$ and which was on par with turmeric rhizome powder@ $@$ \% (17.13\%).

\section{At 90 days after storage}

Till 90 days of storage the botanicals like sweet flag rhizome powder@5\% noticed no insect damage and weight loss followed by neem seed kernel powder @ 5\% (1.93\%). Rest of the treatments was noticed weight loss in the ranged of 8.11 to 20.79 per cent as against 26.71 per cent in untreated check.

\section{At 120 days after storage}

Seed weight loss was maximum in untreated check $(35.24 \%)$ followed by turmeric rhizome powder @ 5\% (26.98\%) and tulsi leaves powder @ 5\% (26.69\%), karanj leaves powder $5 \%(23.65 \%)$. No weight loss of seeds was observed in seeds treated with sweet flag rhizome powder @ 5\% and significantly less weight loss was noticed in neem seed kernel powder @ 5\% (2.89\%). The botanicals i.e. karanj seed powder 5\%, neem leaves powder 5\%, custard apple leaves powder 5\% which was recorded $12.93,15.18$ and 18.53 per cent weight loss, respectively.

\section{At 150 days after storage}

Sweet flag rhizome powder @ 5\% was found superior botanical with no weight loss. The next best treatment in this respect was neem seed kernel powder @ 5\% (4.16\%). Other seed protectants was recorded weight loss in the ranged from 15.85 to 35.85 per cent as against untreated check $(41.90 \%)$.

\section{At 180 days after storage}

At 180 days of after storage, sweet flag rhizome powder @ $5 \%$ protected seeds without any weight loss followed by neem seed kernel powder@5\% (4.73\%). Next treatments viz., karanj seed powder $5 \%$, neem leaves powder $5 \%$, custard apple leaves powder 5\% and karanj leaves powder 5\% found seed weight loss was $18.67,20.07,26.13$, and 34.79 per cent over a period of 6 months. Whereas, highest weight loss was recorded in untreated check $(47.33 \%)$.

\section{Mean weight loss due to $C$. chinensis over 180 days of storage}

The botanicals in reducing the mean weight loss caused by $C$. chinensis in order of their efficacy was sweet flag rhizome powder@5\% (0.00\%)> neem seed kernel powder@5\% $(2.61 \%)>$ karanj seed powder $5 \%(10.19 \%)>$ neem leaves powder $5 \%(11.52 \%)>$ custard apple leaves powder $5 \%$ $(14.14 \%)>$ karanj leaves powder $5 \%(18.55 \%)>$ tulsi leaves powder@5\% (21.97\%) > turmeric rhizome powder@5\% $(24.21 \%)>$ untreated check $(29.88 \%)$.

The present findings are supported with Khalequzzaman et al. (2009) ${ }^{\text {[9] }}$ who reported that no significant weight loss was obtained in $A$. indica seed kernel treated seeds for both bruchid species i.e. C. maculatus and $C$. chinensis. They also reported that highest percentage of weight loss was found in $P$. hydropiper leaf powder followed by A. indica bark, A. squamosa leaf and $V$. negundo leaf powder. Rajasri et al. (2012) ${ }^{[11]}$ found all the neem formulations to be effective against $C$. chinensis in stored blackgram up to 15 months of storage. Hossain and Haque (2010) ${ }^{[6]}$ revealed that all the tested leaf and seed extracts except methi were found effective significantly to check the pulse beetle, $C$. chinensis 
oviposition, adult emergence, seed infestation and weight loss as compared to control on chickpea seeds.

\section{iv) Seed germination}

\section{At 30 days after storage}

All the botanicals maintained germination above Indian Minimum Seed Certification Standard (IMSCS) i.e. 85 per cent except tulsi leaves powder 5\% (84.67\%) turmeric rhizome powder@ $@$ \% (79.00\%) when treated seed stored for 30 days. While, significantly less germination was noticed in untreated check $(75.00 \%)$.

\section{At 60 days after storage}

Seeds of chickpea recorded higher percentage of germination when they were treated with neem seed powder @ 5\% $(91.67 \%)$ followed by sweet flag rhizome powder @ 5\% $(91.33 \%)$, neem leaves powder 5\% $(88.67 \%)$, karanj leaves powder powder 5\% (85.67\%) which was above IMSCS i.e. 85 per cent. Rest of the botanicals was recorded germination in the range of 84.67 to 73.00 percent over untreated check $(64 \%)$.

\section{At 90 days after storage}

All the plant product treatments not maintained germination above IMSCS i.e. 85 per cent except sweet flag rhizome powder@5\% (88.67\%). Whereas, minimum (58.33\%) germination was recorded in untreated check.

\section{At 120 days after storage}

Sweet flag rhizome powder @ 5\% continued to be superior over other botanicals in protecting seeds from $C$. chinensis damage in significantly higher germination $(86.67 \%)$. Rest of the treatments was recorded germination ranged from 80.33 to 61.00 per cent as against untreated check $(56.00 \%)$.

\section{At 150 days after storage}

Among all botanicals germination of chickpea seed was maximum in sweet flag treated seeds with IMSCS i.e.85.67 per cent. Other botanicals was recorded germination below 77.67 per cent over untreated check $(50.33 \%)$.

\section{At 180 days after storage}

The toxicity of all botanicals decreased over a period of 180 days of storage and varied among them, sweet flag rhizome powder@ $@ 5 \%$ was found superior in protecting seeds from $C$. chinensis by recording significantly maximum viability $(85.67 \%)$ and next best botanical treatment was neem seeds kernel powder @ 5\% (76.67\%) at 160 DAS. Rest of the treatments viz., karanj seed powder $5 \%$, neem leaves powder $5 \%$, custard apple leaves powder 5\%, karanj leaves powder $5 \%$ and tulsi leaves powder 5\% was recorded germination of $62.67,62.00,58.67,53.00$ and 52.00 per cent, respectively. The per cent germination was significantly minimum in untreated check $(37 \%)$ followed by turmeric rhizome powder @ 5\% (44.67\%).

\footnotetext{
Mean per cent germination due to $C$. chinensis over 180 days of storage

Over 180 days of storage, mean germination of chickpea seeds in order efficacy of botanicals was sweet flag rhizome powder@\% (88.78\%) > neem seeds kernel powder@5\% $(84.00 \%)>$ karanj seed powder@5\% (77.39\%) > neem leaves powder @ 5\% (77.11\%) > custard apple leaves powder @ 5\% $(73.83 \%)>$ karanj leaves powder@ @ 5 (72.00\%) >
}

tulsi leaves powder @ 5\% (68.22\%) > Turmeric rhizome powder@ @\% (62.78\%) > untreated check (56.78\%).

These results are supported with the results of Tesema et al. $(2015)^{[16]}$ found all the locally available treatment (cow dung ash, leaf powder of neem and leaf powder of basil) were to be effective in reducing the damage inflicted by bruchid, C.chinensis compared to the control in chickpea and other stored legume which is known to cause significant yield loss both quantitatively and qualitatively at monthly up to six months. In the bruchid infested treatment (control), seed germination decreased through time while levels of infestation and weight loss increased. Hossain and Haque (2010) ${ }^{[6]}$ revealed that the extracts of neem seed had no adverse effects on seed germination up to three months of storage studied when infestation caused by pulse beetle, $C$. chinensis.

\section{References}

1. Anonymous. Project Coordinator's Report. AICRP on Chickpea, IIPR, Kanpur 2020.

2. Govindan K, Nelson SJ. Effect of mixtures of plant powder against pulse beetle, Callosobruchus maculatus (F.) (Coleoptera: Bruchidae). Journal of Plant Protection and Environment 2008;5:52-57.

3. Gomez AA, Gomez AA. Statistical procedures for Agriculture Research, New York, Wiley 1984, pp272356.

4. Grainge M, Ahmed S. Handbook of Plants with pest control Properties. New York: John Wiley and sons 1988.

5. Gupta DS, Kashyap RS. Relative resistance of some important varieties of Bengal gram to pulse beetle, Callosobruchus chinensis. Linn. Journal of Research, Punjab Agricultural University 1971;8(3):335-338.

6. Hossain MA, Haque MA. Efficacy of some indigenous plant extracts as grain protectant against pulse beetle, Callosobruchus chinensis L. Journal of Agro- forestry and Environment 2010;4(1):197-202,

7. Hulse SH. Nature, composition and utilization of grain legumes, P. 11-27. In uses of tropical legumes: Proceedings of a consultant meeting 27-30 March, 1989. ICRISAT Centre Patancheru, AP India 1991, 302-324.

8. Kaur, Kamal Deep, Verma SC, Sharma PL. Efficacy of plant powders against pulse beetle, Callosobruchus chinensis, L. infesting pea seed. Journal of Entomology and Zoology Studies 2019;7(1):737-741.

9. Khalequzzaman M, Mahdi SHA, Goni SHMO. Efficacy of edible oils in the control of pulse beetle, Callosobruchus chinensis L. in stored pigeonpea. Rajshahi University. University Journal of Zoology 2009;26:89-92.

10. Metcalf RL, Luckman W. (eds.) Introduction to insect pest management. Willey Inter Science, New York 1975, pp235-273.

11. Rajasri M, Sudharani M, Rao PS. An eco-friendly management of pulse beetle, Callosobruchus chinensis using neem formulations in black gram. International Journal of Science and Research 2012;3:358.

12. Raina AK. Callosobruchus chinensis infesting stored pulses (grain legumes) in India and a comparative study of their biology. Indian J Ent 1970;32:303-310.

13. Sano I. Density effect and environmental tempreture as the factor producing the active form of $C$. maculatus. $\mathrm{J}$ Stored Product Res 1967;2(3):187-195.

14. Shukla R, Kumar A, Prasad CS, Srivastava B, Dubey NK. Efficacy of Acorus calamus L. leaves and rhizome 
on mortality and reproduction of Callosobruchus chinensis L. (Coleoptera: Bruchidae). Applied Entomology and Zoology 2009;44:241-247.

15. Srinivasan T, Durairaj C. Reaction of green gram Accessions Against Bruchid, Callosobruchus maculatus. Indian Journal of Plant Protection 2007;35(2):248-250.

16. Tesema K, Henok Kurabachew, Teferra, Fikre Tadesse. Evaluation of the efficacy of plant powders, cow dung ash, Malathion dust against Callasobruchus chinnsis L (Coleoptera: Bruchidae) on chickpea in Jole Andegna: Southern Ethiopia. Journal of Agricultural Studies 2015;3(2):129-144.

17. Tripathi, Sharda, Sangeeta A, Chandralekha K. Effect of neem leaf powder on infestation of the pulse beetle Callosobruchus chinensis in stored pigeon pea (Cajanus cajan). Vegetos 2006;19(2):27-29. 